\title{
All-photoplastic microstencil with self-alignment for multiple layer shadow-mask patterning
}

\author{
Gyuman Kim ${ }^{\mathrm{a}, *}$, Beomjoon Kim ${ }^{\mathrm{b}}$, Jürgen Brugger ${ }^{\mathrm{a}, 1}$ \\ ${ }^{a}$ NanoLink Strategic Research Orientation, MESA+ Research Institute, University of Twente, \\ P.O. Box 217, 7500 AE Enschede, The Netherlands \\ ${ }^{\mathrm{b}}$ CIRMM, Institute of Industrial Science, University of Tokyo, 4-6-1 Komaba, Meguro-ku, Tokyo 153-8505, Japan
}

Received 12 June 2001; received in revised form 7 May 2003; accepted 4 June 2003

\begin{abstract}
A rapid and simple method to fabricate tiny shadow-masks and their use in multi-layer surface patterning with in situ micromechanical alignment is presented. Instead of using silicon micromachining with through-wafer etching to define the thin membrane with etched apertures, we are using photoplastic SU-8-based resist as structural material of both membrane and support rim. Two layers, 5 and $150 \mu \mathrm{m}$ thick, are structured by lithography and finally released from the surface. The free-standing SU-8 membranes have apertures ranging from 6 to $300 \mu \mathrm{m}$. They are placed and mechanically fixed to the surface, which needs to be patterned. Deposition by evaporation of Cr, Au, $\mathrm{Al}$ or other material through the membrane apertures results in an accurate 1:1 replication of the aperture pattern. In view of multi-layer patterning, we used in situ micromechanical alignment pins or jigs and achieved an overlay precision of $<2 \mu \mathrm{m}$ in both $x$-and $y$-directions. The reusable shadow-masks allows for a low-cost surface patterning technique without the need for photolithography related process steps. It allows unconventional surfaces to be patterned in a rapid and vacuum-clean way on arbitrary surfaces.
\end{abstract}

(C) 2003 Elsevier B.V. All rights reserved.

Keywords: Shadow-mask; Photoplastic; Membrane; Multi-layer evaporation

\section{Introduction}

Surface patterning and MEMS techniques rely on photolithography and thin film processing to form micro-/nanostructures. Some classes of materials and some surfaces are incompatible with resist spinning and associated chemical or thermal process steps. Materials that are difficult or impossible to structure are, for instance, unetchable metals and oxides, biomaterials, gels, polymers, low molecular weight organic and organometallic species. Also surfaces containing fragile micromechanical membranes and cantilevers as often used in deflection sensor or resonator devices cannot be patterned by spin coating processes. Risk of crosscontamination or mechanical damage is the main reason why standard lithography cannot be used. In the growing

\footnotetext{
* Corresponding author. Present address: School of Mechanical Engineering, Kyungpook National University, Daegu 702-701, South Korea. Tel.: +82-53-950-7570; fax: +82-53-950-6550. E-mail address: gyuman.kim@knu.ac.kr (G. Kim).

${ }^{1}$ Present address: Microsystems Laboratory, Ecole Polytechnique Fédérale de Lausanne (EPFL), BM 3116, 1015 Lausanne-Ecublens, Switzerland.
}

field of miniaturized micro- and nanosystems with a variety of three-dimensional devices and chemically functionalized surfaces in biological and lab-on-the-chip applications [1-3], further advancement of soft patterning methods is required to enable the micro- and nanostructuring of technologically challenging but relevant surfaces. Microcontact printing, which uses replicated elastomeric stamps to deliver organic molecules directly and locally onto the surfaces without lithography, allows for short processing cycles and for low-cost pattern replication even at sub-micron scale [4]. Since it relies on a mechanical contact with the surface to transfer the ink, it is not applicable without restriction to an arbitrarily fragile surface.

A truly 'contact-less', hence soft patterning method is area-selective deposition through shadow-masks or stencils. The technique has gained recently great interest as unconventional patterning because it allows forming micro- and nanopatterns without the need for costly photolithography equipment. Flat shadow-masks made of rigid $[5,6]$ as well as elastomeric [7] membranes have been reported. A silicon micromachined three-dimensional shadow-mask for the micropatterning of deeply recessed surfaces with in situ alignment features has also been described $[8,9]$. 


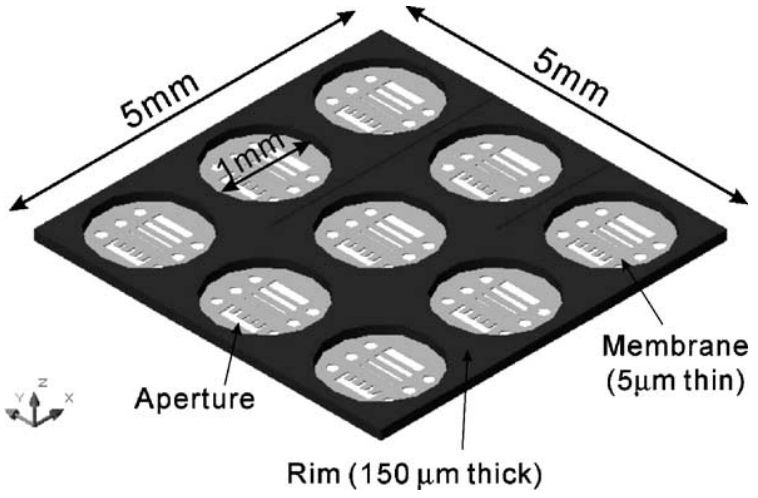

Fig. 1. Schematic view of all-photoplastic shadow-mask. A $5 \mu \mathrm{m}$ thick SU-8 membrane with aperture pattern is sustained by a $150 \mu \mathrm{m}$ thick rim. Material deposition through such shadow-mask allows the definition of surface patterns without lithography on arbitrary surfaces.

Here, in this paper, we describe the realization and use of novel miniaturized shadow-masks as depicted in Fig. 1, and that is made entirely out of photoresist by means of two simple lithography steps. The process of fabricating the shadow-mask device is therefore extremely simplified, since no silicon etching steps are necessary. We also present multi-layer shadow-mask micropatterning having in situ aligning with $<2 \mu \mathrm{m}$ pattern overlay precision.

\section{All-photoplastic shadow-mask}

The miniaturized shadow-mask device consists of two layers of the photoplastic material EPON SU-8 [10-12]. The actual shadow membrane with the apertures patterns is $5 \mu \mathrm{m}$ thick, and the mechanical rim, which sustains the membrane, is $150 \mu \mathrm{m}$ thick. SU-8 is a suitable material for this multi-step microdevice because several layers having different thicknesses $(0.5-200 \mu \mathrm{m})$ can be stacked up by multiple spinning and near-UV light exposure to define the high aspect ratio structures. Cross-linked SU-8 is known to have high residual stress, which causes free-standing membranes to buckle and cracks to appear at the vicinity of sharp corners. In order to reduce the stress in our situation, we have utilized a chemically modified polymer [13]. The composition of both the polymeric binder and the photoacid generator was changed which results in a drastic reduction of the internal stress that occurs typically during the thermal steps. In comparison to unmodified SU-8, stress values of the modified resists results in a stress reduction of $70 \%$. This is an important improvement in order to be able to approach the membrane closely to a flat surface for accurate pattern definition.

Fig. 2 shows details of the shadow-mask microfabrication. First, a release layer is formed on the substrate to allow removal of the shadow-mask from the substrate after fabrication. We have used two release method: first, a $1 \mu \mathrm{m}$ thick layer of $\mathrm{SiO}_{2}$ coated by physical vapor deposition (PVD) (a)

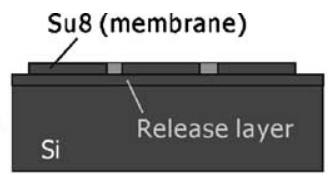

(b)

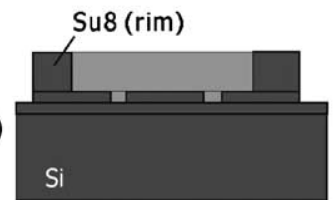

(c)

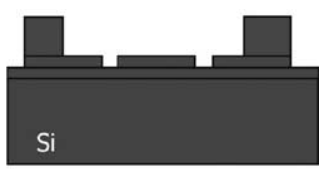

(d)

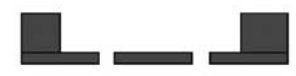

$\mathrm{Si}$

Fig. 2. Schematic outline of all-photoplastic SU-8 shadow-mask fabrication: (a) spinning and exposure of thin membrane; (b) spinning and exposure of thick rim; (c) simultanous development of thin and thick layers; (d) releasing from the surface.

which will serve as sacrificial layer to be selectively removed in $50 \%$ hydroflouric acid (HF) at the end of the process, and second, a 1-2 nm thick organic self-assembled monolayer (SAM) of dodecyltrichlorosilane (DTS) that forms a de-adhesion coating and allows the final SU- 8 device to be removed mechanically from the surface [14]. The advantage of the SAM method is that features with nanometer-scale precision can be replicated into the SU-8 and no wet chemical release step is required. The advantage of the sacrificial PVD $\mathrm{SiO}_{2}$ layer is that very thin and fragile membranes, which would not withstand mechanical lift-off, can be released.

Both methods have been performed successfully by using identical process steps for defining the shadow-mask device with the exception of the final releasing. First, a $5 \mu \mathrm{m}$ thick SU-8 layer is spun on the sacrificial layer, and exposed. Then, a $150 \mu \mathrm{m}$ thick layer is spun and exposed as shown in Fig. 2a and b, respectively. After the simultanous development of the thin and thick layers, the fabricated shadow-mask is lifted off the substrate using one of the previously described releasing steps. In our case, the apertures in the $5 \mu \mathrm{m}$ thick membrane have various shapes and range from 6 to $40 \mu \mathrm{m}$ in width. The handling and support chip is $5 \mathrm{~mm} \times 5 \mathrm{~mm}$ large and has nine circular shadow-mask membranes with $1 \mathrm{~mm}$ in diameter each (Fig. 1).

Fig. 3 shows two scanning electron microscopy (SEM) images of membrane features on bottom side. Fig. 3a shows free-standing finger-like SU-8 structures used for alignment accuracy measurements. The tip of a $40 \mu \mathrm{m}$ long and $5 \mu \mathrm{m}$ thick lever is deflected by $\sim 4 \mu \mathrm{m}$, indicating residual stress of $\sim 2 \mathrm{MPa}$ in the interfacial surface [15]. Fig. 3b shows ray-like apertures also nicely exhibiting the stress in the membrane. Membrane cracking is not observed, which is a clear improvement compared to structures made in earlier experiments using unmodified SU-8 polymer. Further reduction of residual stress can be done by improved thermal budget during processing and by choosing appropriate intermediate layers to minimize the mismatch of thermal expansion coefficients between Si and SU-8 $\left(2.6 \times 10^{-6}\right.$ and $52 \times 10^{-6} \mathrm{~m} / \mathrm{mK}$, respectively). 


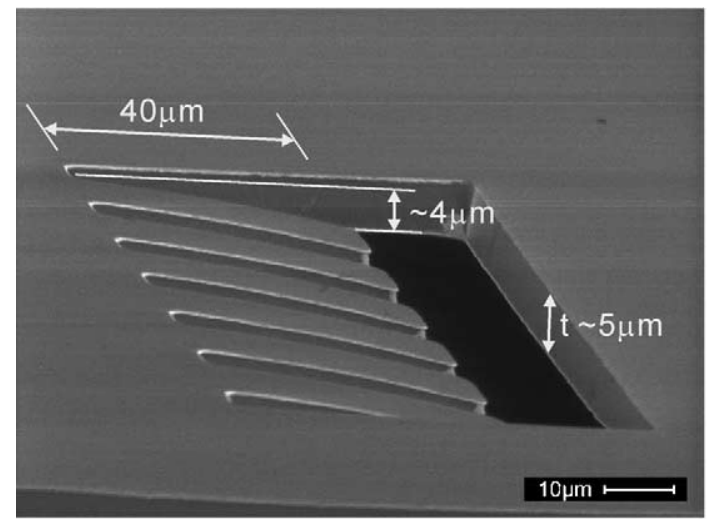

(a)

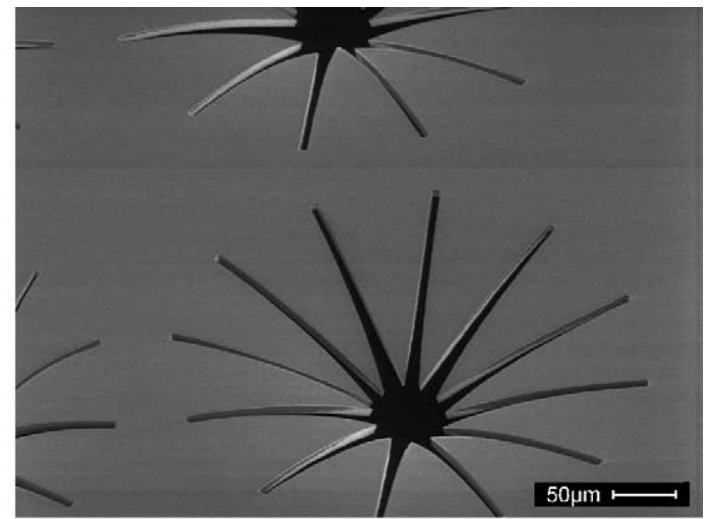

(b)

Fig. 3. (a and b) Scanning electron microscope images of typical membrane structure as seen from the bottom side. No cracks in the sharp corner of aperture are visible. However, bending of membrane still exists in the free-standing cantilevers indicating residual stress.

\section{Multi-layer micropatterning}

\subsection{Alignment of shadow-mask}

Shadow-masks are often used in single-step thin film patterning where alignment to a previously structured layer is not needed. The interest to define several material patterns that are well aligned to each other is however growing, since fully functional devices could be made without the need for lithography techniques, simply by adding structured films, layer by layer. In principle, alignment of shadow-masks can be done on a mask or bond aligner or in situ by using built-in mechanical cavities $[8,16]$.

High aspect ratio SU-8 is well suited for in situ mechanical alignment, and we concentrate here on two approaches as shown in Fig. 4. In both cases, alignment posts are pre-fabricated on the surface that is to be patterned. Since the fabrication of the alignment post is integral part of the shadow-mask fabrication, it serves as an ideal reference structure for the auto-alignment. Both vertical and horizontal alignment techniques by etched pyramid and $\mathrm{V}$-grooves or jig structures are used here. In the case of the etched pyra-

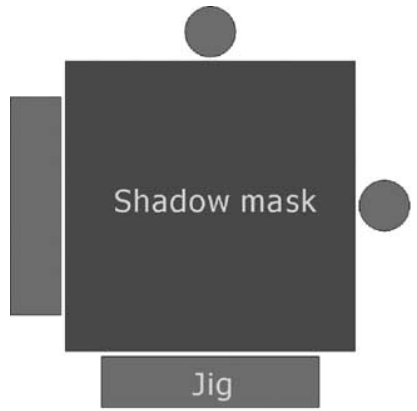

(a)

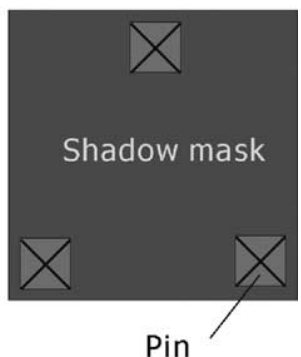

(b)
Fig. 4. Schematic illustration (top view) of two possible alignment methods for multi-layer shadow-mask patterning: (a) mechanical jig structure; (b) alignment pins using $\mathrm{V}$-grooves or pyramids. mid, a complementary structure is directly replicated on the lower side of the shadow-mask. In the other case, we used a jig structure made by SU-8. The positioning and fixing principle is similar to a mechanical jig, which is widely used for alignment during mechanical precision machining [17]. Prior to the evaporation, the shadow-mask is coarse aligned under an optical microscope and then fine aligned using the mechanical posts using the defined reference lines and fixing points in two directions. Finally, it is fixed on the substrate using small springs or tape, which allows it to be removed after evaporation and to be re-used again.

\subsection{Multi-layer deposition with alignment through SU-8 microstencil}

To demonstrate the micropatterning through photoplastic shadow-masks and to analyze the alignment accuracy, we
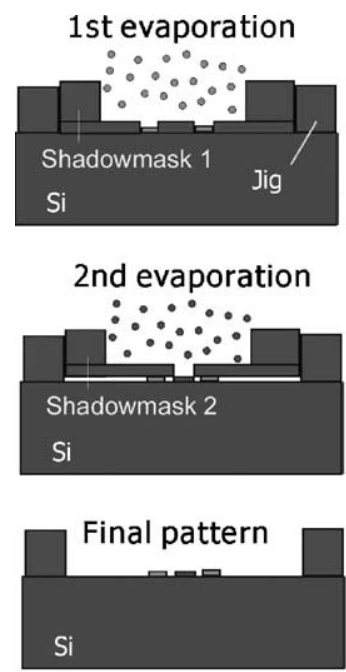

(a)

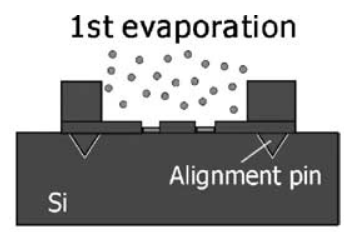

2nd evaporation

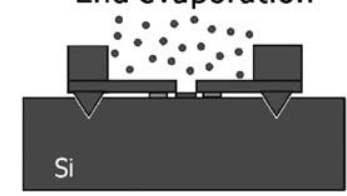

Final pattern

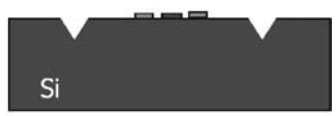

(b)
Fig. 5. Side view of two-layer patterning using two complementary shadow-masks. The alignment is done via: (a) the mechanical jig structure; (b) a previously etched V-groove. 

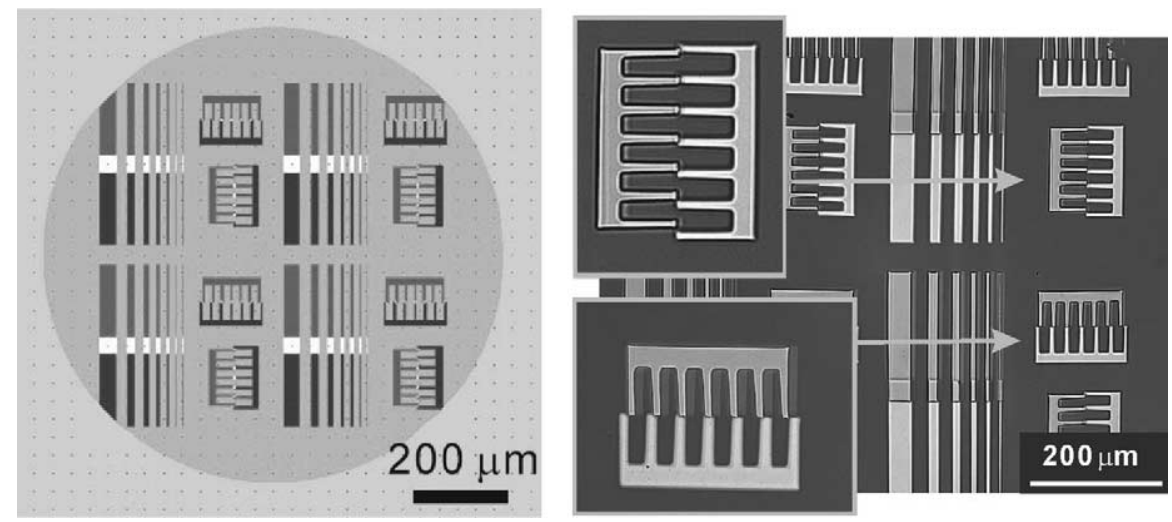

Fig. 6. Schematic picture of shadow-mask design (left) and corresponding results of alignment test structure in two metal layers (Al and Au) (right). The pattern was evaporated using the jig alignment scheme. A precision of $<2 \mu \mathrm{m}$ could be achieved in $x$ - and $y$-directions.

performed two subsequent area-selective deposition steps of two metals through two shadow-masks with complementary patterns.

Fig. 5 shows schematically the deposition of the two layers with mechanically aligned shadow-masks. By evaporation, first a $100 \mathrm{~nm}$ thick aluminum $(\mathrm{Al})$, and second a $200 \mathrm{~nm}$ thick gold $\mathrm{(Au}$ ) layer were deposited. The base pressure during evaporation is $3 \times 10^{-4} \mathrm{~Pa}$, and the distance between the metal evaporation source and the substrate surface is $300 \mathrm{~mm}$. Figs. 6 and 7 show typical Al/Au pattern. The test pattern (Fig. 6) shows an alignment accuracy in $x$ - and $y$-directions of $\sim 2 \mu \mathrm{m}$. The smallest line width so far obtained is $4.5 \mu \mathrm{m}$, which is $\sim 1.5 \mu \mathrm{m}$ smaller than the mem- brane aperture width. We attribute this width loss to side-wall shadowing effect as result of the membranes thickness and oblique incident angle of the evaporated material. Other test structures are shown in Fig. 7. In principle, arbitrary patterns with the exception of closed rings (doughnut problem) can be made at various length scales and with micrometer overlay precision without lithography on the substrate surface.

A closer look at the structures in Fig. 7 shows mirror structures that are slightly shifted with respect to the $\mathrm{Au}$ pattern. They are originating from the $10 \mathrm{~nm}$ chrome $(\mathrm{Cr})$ adhesion coating, which is done before the Au evaporation from a differently positioned source. The $10 \mathrm{~nm} \mathrm{Cr}$ and the

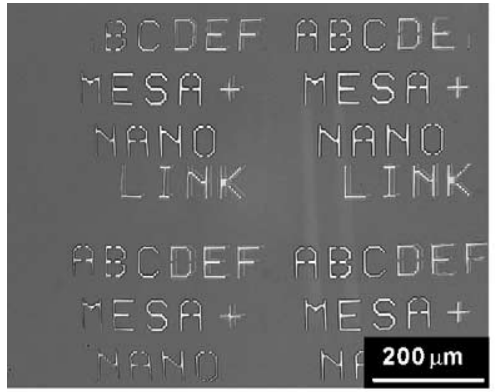

(a)

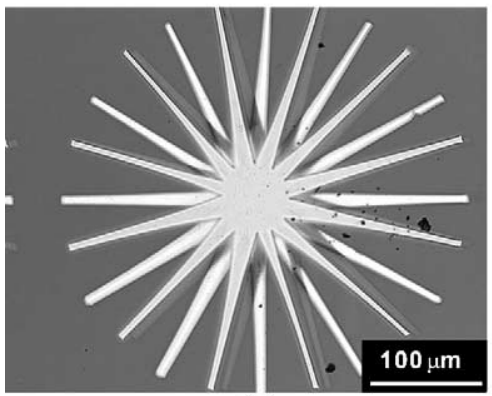

(c)

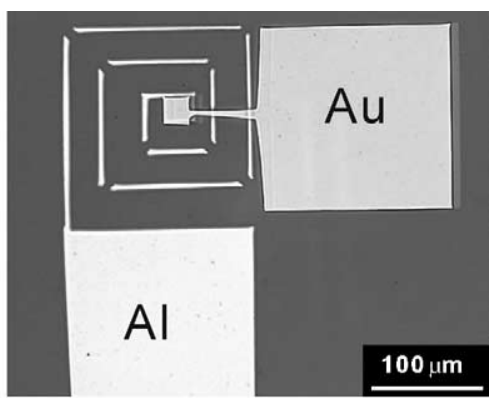

(b)

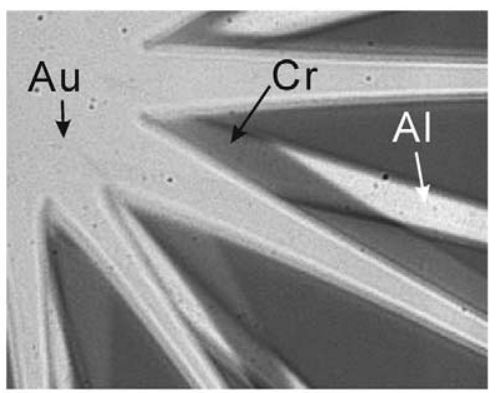

(d)

Fig. 7. Other multi-layer patterns of $\mathrm{Al}, \mathrm{Cr}$ and $\mathrm{Au}$ demonstrating the in situ alignment capability: (a) micron-scale letters; (b) aligned micro-lines with large contact pad; (c) double-star pattern of $\mathrm{Al}$ and $\mathrm{Cr} / \mathrm{Au}$; (d) enlarged image of double-star pattern. Chromium and gold are deposited subsequently through the same shadow-mask, but due to the different source positions and a small membrane-to-surface gap, the Au pattern is laterally shifted. 
$200 \mathrm{~nm}$ Au layer are not deposited exactly on the same position as shown in detail in Fig. 7d. This affect can actually be utilized to form closely spaced micro-/nanopatterns without having to remove the shadow-mask [18].

During the fabrication of the SU-8 shadow-mask, the difference of thermal expansion between the polymer and the Si substrate causes residual stress in the layers, which result in membrane buckling after its removal from the surface. The effect is that the shadow-mask cannot be approached perfectly to a flat surface over a large area. The warping of the $5 \mu \mathrm{m}$ thick membrane can be as large as several tens of microns, depending on the size of the free-standing features, resulting in a similar gap between shadow-mask and substrate. For large $(>100 \mu \mathrm{m})$ features, this effect can be neglected, but it was clearly observed for small-sized $(<20 \mu \mathrm{m})$ patterns. At small scale, the membrane warping affects the alignment accuracy as well as pattern shape. Therefore, further process improvement in using low-stress polymer resists is necessary to fabricate flat low-stress free-standing SU-8 membranes.

\section{Conclusion}

A new method for making free-standing photoresist membranes is shown that can be patterned as miniature shadow-masks. It allows speeding up the cycle from design to material micropatterns since no silicon micromachining technique is necessary, and since it allows for pattern formation without lithography steps. Shadow-mask techniques are promising to pattern unconventional materials on unconventional surfaces since it eliminates all potential chemically induced cross-contamination as well as mechanical damage of fragile functionalized substrates. Arbitrarily shaped multi-layer $\mathrm{Au} / \mathrm{Al}$ patterns as small as $4.5 \mu \mathrm{m}$ were defined with a $2 \mu \mathrm{m}$ alignment accuracy to each other using on-chip mechanical alignment structures. The mechanical properties of the SU-8 resist paired with an extremely simple fabrication process of thin membranes together with thick handle and support rims is a major advantage over the conventional silicon micromachining processes to fabricate miniature shadow-masks, which can be used over and over again in environment, where lithography equipment is not readily accessible.

\section{Acknowledgements}

We are pleased to acknowledge many of our MESA+ colleagues for helpful discussions and technical assistance.
We thank specifically 'mrt Berlin' for providing us with modified SU-8-based resist and for helpful discussions. This research is financially supported by the NanoLink Strategic Research Orientation of MESA+ Research Institute, University of Twente, The Netherlands.

\section{References}

[1] M. Madou, Fundamentals of Microfabrication, CRC Press, Boca Raton, and references therein, 1997 (ISBN: 0-8493-9451-1).

[2] H.G. Craighead, Nanoelectromechanical systems, Science 290 (2000) $1532-1535$

[3] A. van den Berg, W. Olthuis, P. Bergveld (Eds.), Micro Total Analysis Systems 2000, Kluwer Academic Publishers, Dordrecht, and references therein, 2000 (ISBN: 0-7923-6387-6).

[4] Y. Xia, G.M. Whitesides, Soft lithography, Angew. Chem. Int. Ed. 37 (1998) 550-575.

[5] S. Fan, M.G. Chapline, N.R. Franklin, T.W. Tombler, A.M. Cassell, H. Dai, Self-oriented regular arrays of carbon nanotubes and their field emission properties, Science 283 (1999) 512-514.

[6] J. Brugger, J.W. Berenschot, S. Kuiper, W. Nijdam, B. Otter, M. Elwenspoek, Resistless patterning of sub-micron structures by evaporation through nanostencils, Microelectron. Eng. 53 (2000) 403-405.

[7] R.J. Jackman, D.C. Duffy, O. Cherniavskaya, G.M. Whitesides, Using elastomeric membranes as dry resists and for dry lift-off, Langmuir 15 (1999) 2973-2984.

[8] J. Brugger, C. Andreoli, M. Despont, U. Drechsler, H. Rothuizen, P. Vettiger, Self-aligned 3D shadow mask technique for patterning deeply recessed surfaces of micro-electro-mechanical systems devices, Sens. Actuators 76 (1999) 329-334.

[9] US Patent no. 6,080,513 (4 May 1998).

[10] K.Y. Lee, N. LaBianca, S.A. Rishton, S. Zolgharnain, J.D. Gelorme, J. Shaw, T.H.P. Chang, Micromachining applications of a high resolution ultrathick photoresist, J. Vac. Sci. Technol. B 13 (1995) 3012-3016.

[11] M. Despont, H. Lorenz, N. Fahrni, J. Brugger, P. Renaud, P. Vettiger High-aspect-ratio, ultrathick, negative-tone near-UV photoresist for MEMS applications, Proc. MEMS 1997 (1997) 518-522.

[12] H. Lorenz, M. Despont, N. Fahrni, J. Brugger, P. Vettiger, P. Renaud, High-aspect-ratio, ultrathick, negative-tone near-UV photoresist and its applications for MEMS, Sens. Actuators A 64 (1998) 33-39.

[13] R. Ruhmann, G. Ahrens, A. Schuetz, J. Voskuhl, G. Gruetzner, Reduction of internal stress in a SU-8 like negative tone photoresist for MEMS applications by chemical modification, SPIE Microlithogr. 43 (2001) 45-57.

[14] B.J. Kim, M. Liebau, J. Huskens, D.N. Reinhoudt, J. Brugger, A self-assembled monolayer-assisted surface microfabrication and release technique, Microelectron. Eng. 57 (2001) 755-760.

[15] T. Hubbard, J. Wylde, Residual strain and resultant postrelease deflection of surface micromachined structures, J. Vac. Sci. Technol. A 18 (2000) 734-737.

[16] A. Tixier, Y. Mita, J.P. Gouy, H. Fujita, A silicon shadow mask for deposition on isolated areas, J. Micromech. Microeng. 10 (2000) $157-162$.

[17] E.K. Henriksen, Jig and Fixture Design Manual, Industrial Press Inc., 1973.

[18] G.J. Dolan, Offset masks for lift-off photoprocessing, Appl. Phys. Lett. 31 (1977) 337-339. 